\title{
Remote Sensing of Wetland Flooding at a Sub-Pixel Scale Based on Random Forests and Spatial Attraction Models
}

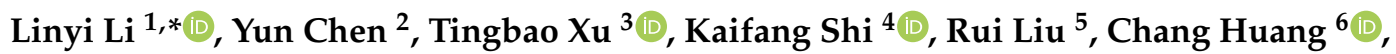 \\ Binbin Lu ${ }^{1}$ and Lingkui Meng ${ }^{1}$ \\ 1 School of Remote Sensing and Information Engineering, Wuhan University, Wuhan 430079, China; \\ binbinlu@whu.edu.cn (B.L.); lkmeng@whu.edu.cn (L.M.) \\ 2 Commonwealth Scientific and Industrial Research Organisation (CSIRO) Land and Water, Canberra 2601, \\ Australia; Yun.Chen@csiro.au \\ 3 Fenner School of Environment and Society, The Australian National University, Canberra 2601, Australia; \\ tingbao.xu@anu.edu.au \\ 4 Chongqing Engineering Research Center for Remote Sensing Big Data Application, School of Geographical \\ Sciences, Southwest University, Chongqing 400715, China; skffyy@swu.edu.cn \\ 5 Beijing Laboratory of Water Resource Security, Capital Normal University, Beijing 100048, China; \\ RuiLiu@cnu.ed.cn \\ 6 College of Urban and Environmental Sciences, Northwest University, Xi'an 710127, China; \\ changh@nwu.edu.cn \\ * Correspondence: lilinyi@whu.edu.cn; Tel.: +86-135-4517-7585
}

Received: 9 May 2019; Accepted: 22 May 2019; Published: 24 May 2019

\begin{abstract}
Wetland flooding is significant for the flora and fauna of wetlands. High temporal resolution remote sensing images are widely used for the timely mapping of wetland flooding but have a limitation of their relatively low spatial resolutions. In this study, a novel method based on random forests and spatial attraction models (RFSAM) was proposed to improve the accuracy of sub-pixel mapping of wetland flooding (SMWF) using remote sensing images. A random forests-based SMWF algorithm (RM-SMWF) was developed firstly, and a comprehensive complexity index of a mixed pixel was formulated. Then the RFSAM-SMWF method was developed. Landsat 8 Operational Land Imager (OLI) images of two wetlands of international importance included in the Ramsar List were used to evaluate RFSAM-SMWF against three other SMWF methods, and it consistently achieved more accurate sub-pixel mapping results in terms of visual and quantitative assessments in the two wetlands. The effects of the number of trees in random forests and the complexity threshold on the mapping accuracy of RFSAM-SMWF were also discussed. The results of this study improve the mapping accuracy of wetland flooding from medium-low spatial resolution remote sensing images and therefore benefit the environmental studies of wetlands.
\end{abstract}

Keywords: wetland flooding; random forests; spatial attraction models; comprehensive complexity index; sub-pixel mapping

\section{Introduction}

Wetlands are among the most diverse and productive ecosystems on the earth, which are created by the impact of prolonged inundation and are characterized by specific soil quality and diverse flora and fauna $[1,2]$. Wetlands are indispensable for many benefits, including freshwater supply, flood control, groundwater recharge, and climate change mitigation [2]. Wetland flooding is necessary for the flora and fauna of wetlands and is important for the recovery of the wetland biodiversity $[1,2]$. 
Remote sensing images with high temporal resolutions have been widely used for monitoring flooding, providing timely flooding distribution information [3-6]. However, these images usually have relatively low spatial resolutions, which limit the mapping accuracy. One of the popular ways to improve mapping accuracy is sub-pixel mapping.

Sub-pixel mapping can be employed to improve the mapping accuracy of wetland flooding from remote sensing images with high temporal resolutions. Sub-pixel mapping can divide a mixed pixel into multiple sub-pixels to separate the mixed information contents and then obtain an accurate spatial distribution ration of the mixed information contents, therefore, improving the mapping accuracy. Various sub-pixel mapping methods have been proposed in recent years, such as methods based on spatial attraction models (SAM) [7-9], artificial neural networks [10,11], Markov random field [12], and swarm intelligence $[13,14]$. However, due to the complexity and uncertainty of remote sensing images with relatively low spatial resolutions, sub-pixel mapping of wetland flooding (SMWF) is still difficult and needs further development.

Among the aforementioned sub-pixel mapping methods, SAM has several advantages in terms of its simplicity and its explicit physical meanings [8]. Spatial attraction is defined in SAM, calculating the spatial correlation between a sub-pixel and its surrounding pixels. SAM does not require training samples and prior knowledge on the spatial structure of mixed pixels. However, SAM does not always obtain satisfactory SMWF results when the situation of the mixed pixels is complex. As a popular artificial intelligence algorithm, random forests (RF) have attracted extensive attention in recent years [15-18]. Random forests are a combination of tree predictors where each tree depends on the values of a random vector sampled independently [19]. Injecting the right kind of randomness makes random forests accurate classifiers [19]. Random forests generate an internal unbiased estimate of the generalization error as the forest building progresses and do not overfit as more trees are added into the forest [20]. Because of their excellent performance in classification and regression, random forests have been applied to many fields, such as environmental science [21-23], medical science [24,25], energy [26-28], traffic [29,30], economics [31], and remote sensing [32-34]. SMWF is a sub-pixel classification issue in essence [5]. The assumption of this study is that the performance of SMWF should be enhanced if SAM is coupled with random forests.

SMWF based on random forests and spatial attraction models (RFSAM) is relatively rare in the literature. In this study, a novel RFSAM-based SMWF (RFSAM-SMWF) method is proposed to improve the mapping accuracy of wetland flooding at a sub-pixel scale from remote sensing images. The main objectives are: (1) to develop an RF-SMWF algorithm, which is the basis of RFSAM-SMWF; (2) to formulate a comprehensive complexity index of a mixed pixel and develop the RFSAM-SMWF algorithm; (3) to compare the results of RFSAM-SMWF to traditional SMWF methods using Landsat 8 Operational Land Imager (OLI) images of two wetlands of international importance included in the Ramsar List; and (4) to discuss the mapping accuracy of RFSAM-SMWF related to the number of trees in random forests and the complexity threshold.

\section{Methods}

\subsection{Principle of SMWF}

The intention of SMWF is to obtain the most likely sub-pixel spatial distributions of wetland flooding within a mixed pixel, while maintaining the original proportion of wetland flooding and maximizing spatial dependence. An illustration of SMWF with two classes representing wetland flooding and non-flooding is shown in Figure 1. A fraction image of wetland flooding is shown in Figure 1a, which is the input to SMWF. The fraction value does not specify the distributions of flooding sub-pixels; hence, there could be many possible compositions of flooding and non-flooding sub-pixels. Figure $1 \mathrm{~b}-\mathrm{d}$ shows three possible flooding distributions in the central mixed pixel. The scale factor is 3 and the fraction value of the central mixed pixel is $55.6 \%$; therefore, there are 5 flooding sub-pixels and 4 non-flooding sub-pixels in the central mixed pixel. According to the spatial dependence, the most 
likely distribution of sub-pixels among the three distributions should be Figure $1 \mathrm{~d}$. That is because the two $100 \%$ flooded pixels nearby give a higher likelihood of flooding by proximity. However, it is usually difficult to find the most likely sub-pixel distributions from numerous possible distributions in the actual SMWF.

\begin{tabular}{|c|c|c|}
\hline $22.2 \%$ & $66.7 \%$ & $100 \%$ \\
\hline $33.3 \%$ & $55.6 \%$ & $100 \%$ \\
\hline $0 \%$ & $22.2 \%$ & $44.4 \%$ \\
\hline
\end{tabular}

(a)

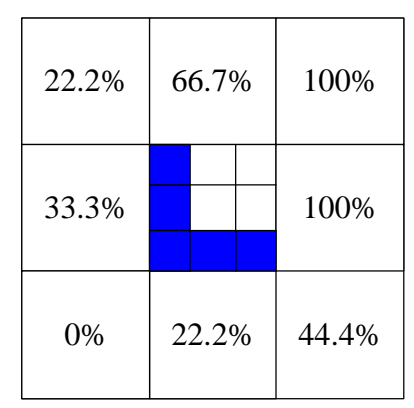

(b)

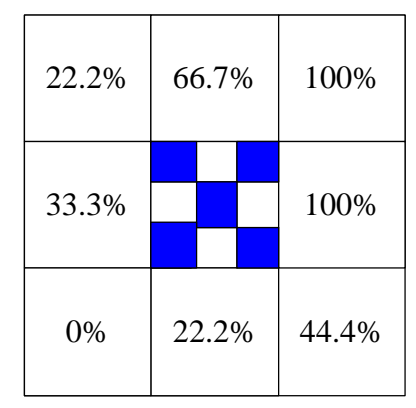

(c)

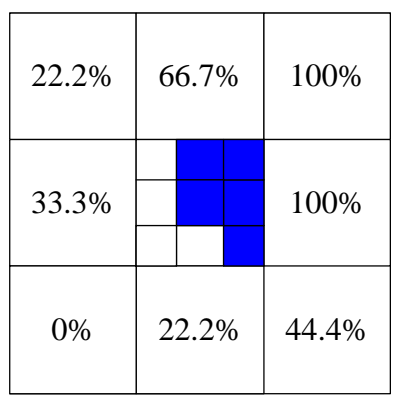

(d)

wetland flooding

non-flooding

Figure 1. An illustration of SMWF (scale factor = 3). (a) Fraction image of wetland flooding; (b) Possible flooding distribution 1. (c) Possible flooding distribution 2. (d) Possible flooding distribution 3.

\subsection{SAM-SMWF Algorithm}

SAM-SMWF uses SAM to obtain the spatial distributions of wetland flooding at a sub-pixel scale. The rationale of SAM is as follows [35]:

(1) SAM is based on the fraction values in neighboring pixels acting towards sub-pixels inside a central mixed pixel.

(2) A sub-pixel can be attracted only by pixels surrounding the central pixel. A maximum of eight neighboring pixels can be considered for attraction.

(3) Other pixels are assumed too distant to exercise the attraction.

The flooding attraction value (FAV) and non-flooding attraction value (NAV) for a sub-pixel $p_{\mathrm{x}, y}$ are calculated as follows [35]:

$$
\begin{gathered}
F A V\left(p_{x, y}\right)=\frac{\sum_{n=1}^{N} F F V(P(n)) / d\left(p_{x, y}, P(n)\right)}{N} \\
N A V\left(p_{x, y}\right)=\frac{\sum_{n=1}^{N}(1-F F V(P(n))) / d\left(p_{x, y}, P(n)\right)}{N} \\
d\left(p_{x, y}, P(n)\right)=\sqrt{[x+0.5-s(i+0.5)]^{2}+[y+0.5-s(j+0.5)]^{2}}
\end{gathered}
$$


where $N$ is the number of the neighboring pixels, $s$ is the scale factor, $\operatorname{FFV}(P(n))$ is the flooding fraction value of the pixel $P(n), d\left(p_{x, y}, P(n)\right)$ is the distance between a sub-pixel $p_{x, y}$ and a neighboring pixel $P(n), i, j$ are the row and column of the neighboring pixel $P(n)$ relatively to the central pixel, respectively, and $x, y$ are the row and column of the sub-pixel $p_{x, y}$ in the central mixed pixel, respectively.

If $F A V\left(p_{x, y}\right) \geq N A V\left(p_{x, y}\right)$, then the sub-pixel $p_{x, y}$ is classified as wetland flooding. Otherwise the sub-pixel $p_{x, y}$ is classified as non-flooding.

\subsection{RFSAM-SMWF Algorithm}

In this study, we first developed an RF-SMWF method as the basis of RFSAM-SMWF. Random forests are a classifier consisting of a collection of tree-structured classifiers [19,20]. While training, a random third of all training samples are excluded from the training set for each tree, called "out-of-bag". The remaining two-thirds generate a single tree. Each descendant node is split from a parent node for the growth of a tree, using a random subset of the input variables. The "out-of-bag" samples are an independent test set for evaluating the tree internally. To classify a new sample, the input vector of the sample is put down each of the trees in the forest. Each tree gives a classification, and the tree "votes" for that class. The forest chooses the classification which has the most votes over all the trees in the forest. SMWF is a sub-pixel classification issue in essence [5]. In RF-SMWF, a set of random forests is trained to describe the relationship between the fractions of the eight neighboring pixels of a mixed pixel and sub-pixels within the mixed pixel. The number of random forests is equal to the number of sub-pixels in a mixed pixel. The input to the set of random forests is the fractions of the eight neighbors of a mixed pixel. The output of the set of random forests is the classification of sub-pixels of a mixed pixel.

RFSAM-SMWF obtains the spatial distributions of wetland flooding at a sub-pixel scale based on random forests and spatial attraction models. The complexity of mixed pixels is computed based on the comprehensive complexity index (CCI) in RFSAM-SMWF. CCI of a mixed pixel considers the complexity of the mixed pixel itself and the complexity of the neighboring pixels of the mixed pixel. It is formulated as follows:

$$
\begin{gathered}
C C I=\left(C I_{S}+C I_{N}\right) / 2 \\
C I_{S}=\operatorname{choose}\left(\mathrm{m}_{,} \mathrm{k}_{\mathrm{s}}\right) / \operatorname{choose}(\mathrm{m}, \operatorname{int}(\mathrm{m} / 2)) \\
C I_{N}=\frac{1}{N} \sum_{n=1}^{N} \operatorname{choose}\left(\mathrm{m}, \mathrm{k}_{\mathrm{n}}\right) / \operatorname{choose}(\mathrm{m}, \operatorname{int}(\mathrm{m} / 2))
\end{gathered}
$$

where $C I_{S}$ represents the complexity index of the mixed pixel itself, $C I_{N}$ represents the complexity index of the neighboring pixels of the mixed pixel, $N$ is the number of the neighboring pixels of the mixed pixel, $\mathrm{k}_{\mathrm{s}}$ is the number of flooding sub-pixels of the mixed pixel, and $\mathrm{k}_{\mathrm{n}}$ is the number of flooding sub-pixels of the neighboring pixel. The function choose $(\mathrm{m}, \mathrm{k})=m ! /((\mathrm{m}-\mathrm{k}) ! \mathrm{k} !)$ is the binomial coefficient, where $\mathrm{m}$ is the number of sub-pixels of a mixed pixel. The function int $(\mathrm{x})$ obtains the nearest integer less than or equal to $x$.

If a mixed pixel is in a complex situation according to CCI, RFSAM-SMWF constructs a RF-SMWF model to obtain the sub-pixel distributions of wetland flooding. Otherwise, RFSAM-SMWF obtains the sub-pixel distributions of wetland flooding using SAM. The situation of a mixed pixel is considered as complex if its CCI value is larger than the complexity threshold. Setting the complexity threshold is therefore critical to the performance of RFSAM-SMWF and is discussed in the Discussion section. A flow chart of RFSAM-SMWF is shown in Figure 2. 


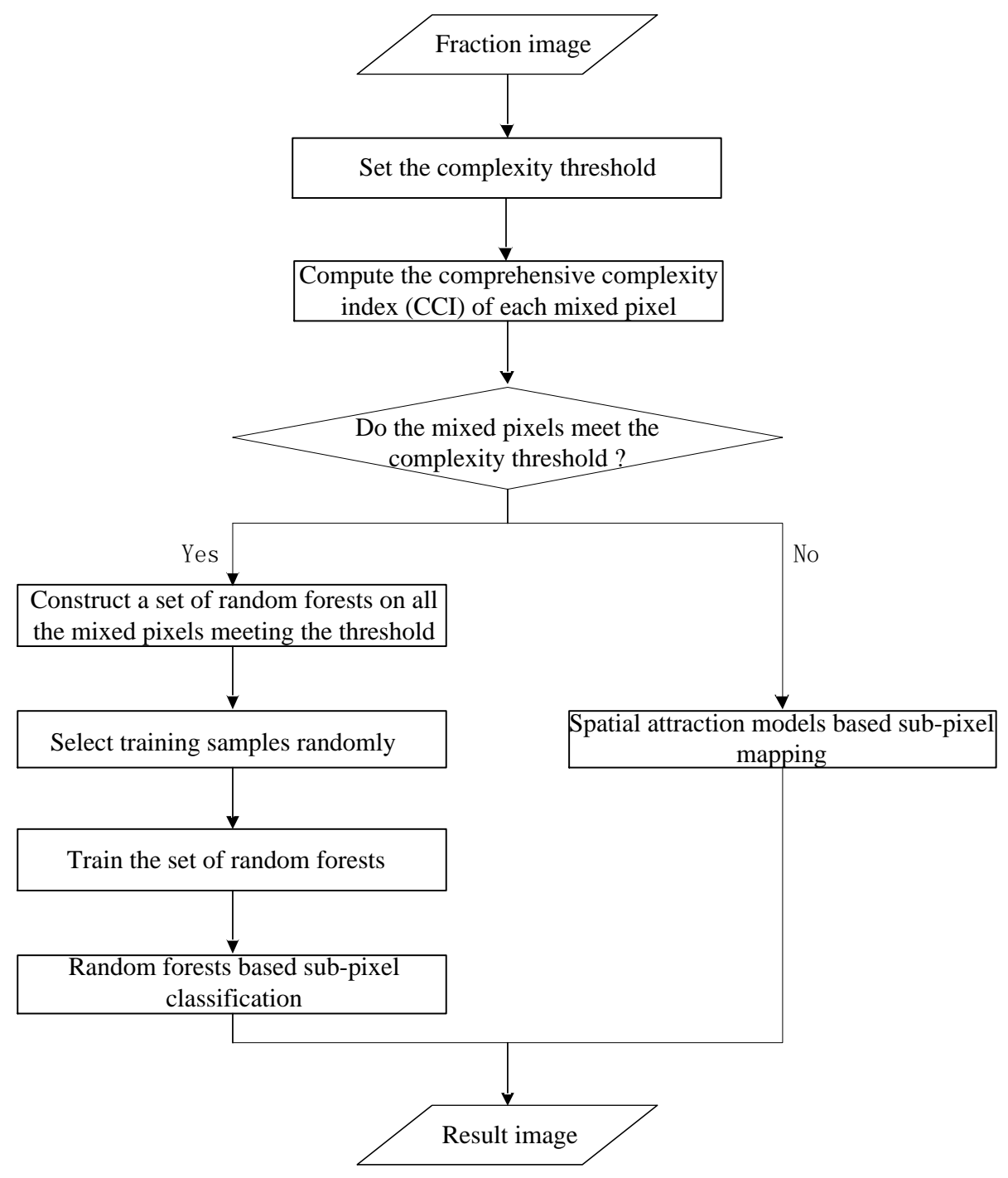

Figure 2. A flow chart of RFSAM-SMWF.

\section{Case Study}

\subsection{Study Materials}

Two comparative study areas were selected from two representative wetlands designated by the Ramsar Convention as wetlands of global importance [36]. The first wetland is East Dongting Lake Wetland, which is located in Hunan Province, China, and was listed on 31 March 1992. The second wetland is Honghu Wetland, which is located in Hubei Province, China, and was listed on 2 February 2008. The two wetlands experienced significant floods in July 2017 and July 2016, respectively. Corresponding Landsat 8 OLI images were obtained for the two wetlands. Key characteristics of the study areas are summarized and shown in Table 1.

Locations of the two study areas are shown in color composite images (R6G3B2) in Figure 3. The flooding reference images were obtained from the corresponding Landsat 8 OLI images using the modified normalized difference water index (mNDWI) [37,38]. The resolution of the flooding reference images was $30 \mathrm{~m}$. The flooding fraction images were obtained by aggregating the corresponding flooding reference images. The scale was set at 5 . Therefore, the resolution of the flooding fraction images was $150 \mathrm{~m}$. The value of the aggregated pixel equaled the proportion of flooding pixels inside the corresponding $5 \times 5$ window. The flooding fraction images were the inputs to the SMWF methods. 
Table 1. Key characteristics of the two comparative study areas.

\begin{tabular}{ccc}
\hline & Study Area 1 & Study Area 2 \\
\hline Location & East Dongting Lake Wetland, Hunan Province, China & Honghu Wetland, Hubei Province, China \\
Ramsar site number & 551 & 1729 \\
Ramsar designation date & 31 March 1992 & 2 February 2008 \\
Experimental data & Landsat 8 OLI imagery & Landsat 8 OLI imagery \\
Image date & 26 July 2017 & 23 July 2016 \\
Image size & $500 \times 500$ pixels & $500 \times 500$ pixels \\
Image resolution & $30 \mathrm{~m}$ & $30 \mathrm{~m}^{2}$ \\
Area & $225 \mathrm{~km}^{2}$ & $225 \mathrm{~km}^{2}$ \\
\hline
\end{tabular}

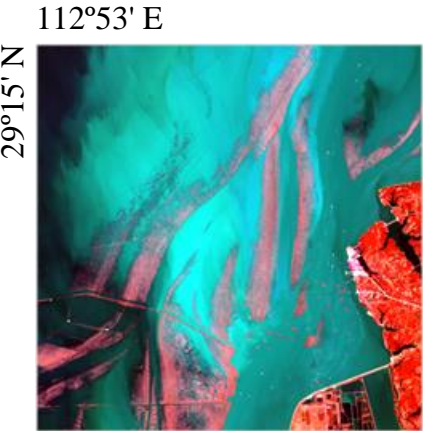

$113^{\circ} 02^{\prime} \mathrm{E}$

Landsat 8 image (R6G3B2)<smiles>C=CC</smiles>

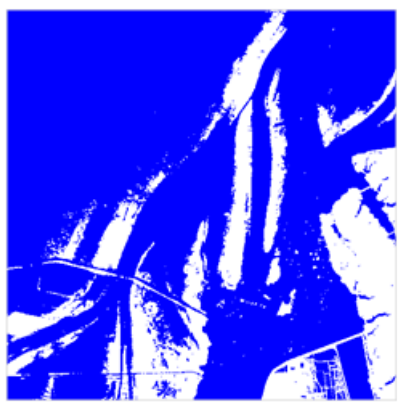

Flooding reference image<smiles>C=CC</smiles>

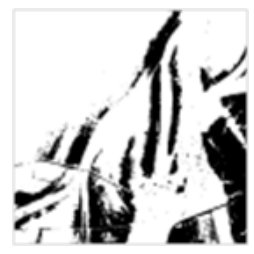

Flooding fraction image

(a)

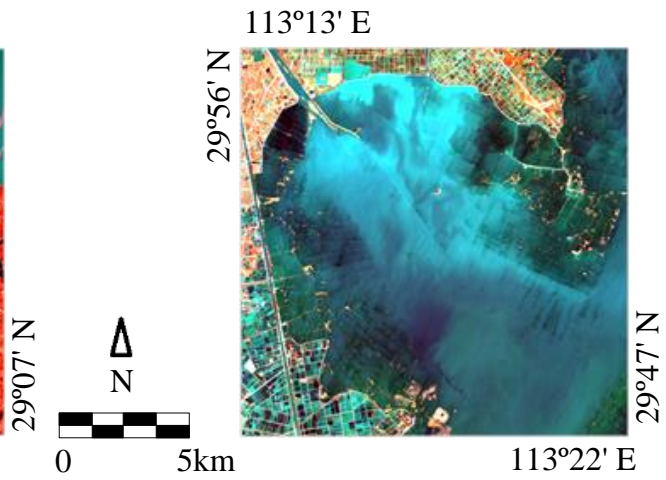

Landsat 8 image (R6G3B2)
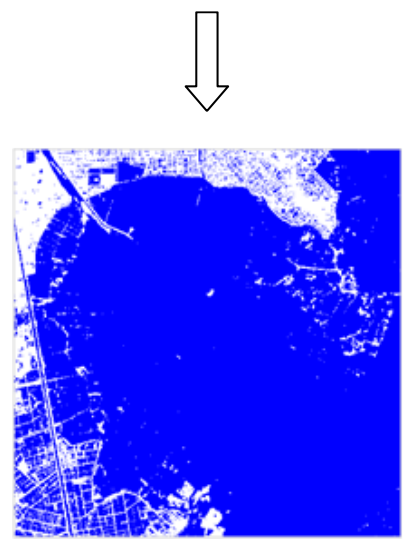

Flooding reference image

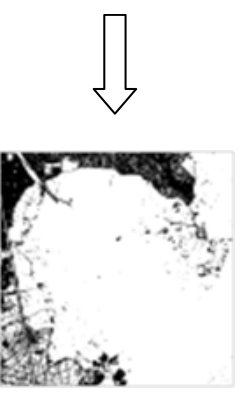

Flooding fraction image

(b)

Figure 3. Experimental images of the two study areas. (a) East Dongting Lake Wetland; (b) Honghu Wetland.

Wetland flooding has its own spatio-temporal characteristics. To apply and verify the RFSAM-SMWF algorithm, ideally, we should have two sets of multispectral remote sensing images 
with different spatial resolutions acquired at the same time over the study area: a low resolution image from which the flooding fraction image would be derived, and another higher resolution image from which the flooding reference image could be obtained. However, although there are many remote sensing satellites orbiting, it was challenging to find valid images from two different satellite sensors at a close enough time in the specific study area. In that case, areas covered by high spatial resolution satellite data would be the primary option since we could obtain a low spatial resolution flooding fraction image by aggregating a higher resolution flooding reference image, but not vice versa. The scale was set at 5 , which was an empirical option that had been proved effective for applying sub-pixel algorithms and had also been adopted by other studies [10,39].

\subsection{Results and Analysis}

The four SMWF methods for comparison are SMWF (BP-SMWF) based on a back-propagation neural network [40], SAM-based [35] SMWF (SAM-SMWF), decision tree-based [40] SMWF (DT-SMWF), and RFSAM-SMWF. The four methods were coded in Matlab. RFSAM-SMWF was coded using the TreeBagger function in Matlab, which implements the random forests algorithm [40]. The number of trees was set at 20 and the complexity threshold was set at 0.1 in RFSAM-SMWF. The same neighboring type of mixed pixels was used for the four methods. Twenty percent of the mixed pixels in the flooding fraction images were randomly selected as training samples for BP-SMWF, DT-SMWF, and RFSAM-SMWF. The hidden layer number was one in BP-SMWF.

Visual comparisons of the four SMWF methods for the two wetlands are shown in Figures 4 and 5. To provide clarity, the same small regions of the reference images and the four SMWF results are zoomed in. As shown in Figure 4, RFSAM-SMWF obtained the best SMWF result for East Dongting Lake Wetland. RFSAM-SMWF integrates SAM with random forests for SMWF and therefore further improves the performance of SMWF. From Figure 5, we can see that RFSAM-SMWF also gave the best visual SMWF result for Honghu Wetland.

The quantitative assessments of the four SMWF methods are shown in Table 2. The results were evaluated based on overall accuracy (OA), Kappa coefficient (KC), average producer's accuracy (APA), and average user's accuracy (AUA) [41,42]. Only mixed pixels in the flooding fraction images were included when calculating the evaluation indices. RFSAM-SMWF obtained the highest evaluation indices for both wetlands. For example, the OA values of BP-SMWF, SAM-SMWF, DT-SMWF, and RFSAM-SMWF are 72.8\%, 75.8\%, 75.5\%, and 80.2\% for East Dongting Lake Wetland, respectively. The OA values of BP-SMWF, SAM-SMWF, DT-SMWF, and RFSAM-SMWF are 70.1\%, 73.3\%, 70.2\%, and $76.7 \%$ for Honghu Wetland, respectively. 

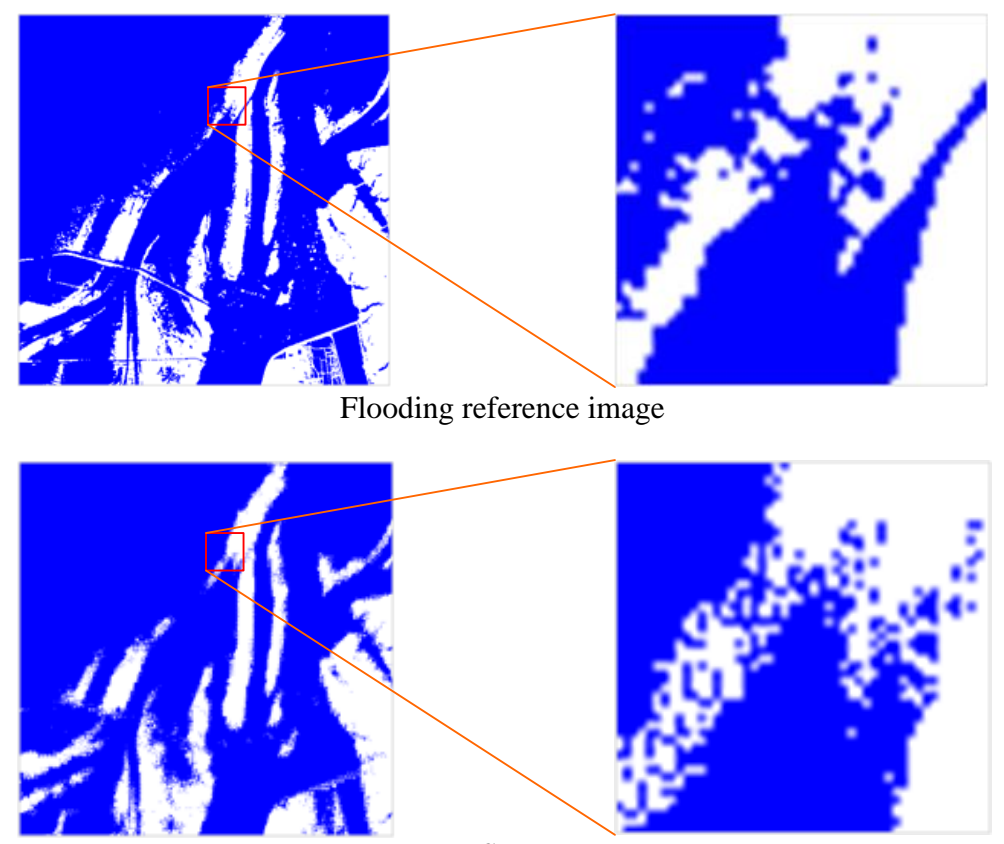

BP-SMWF

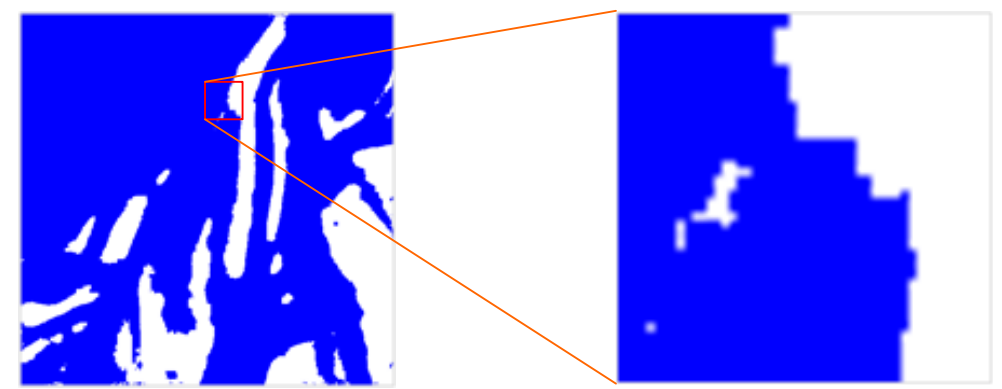

SAM-SMWF

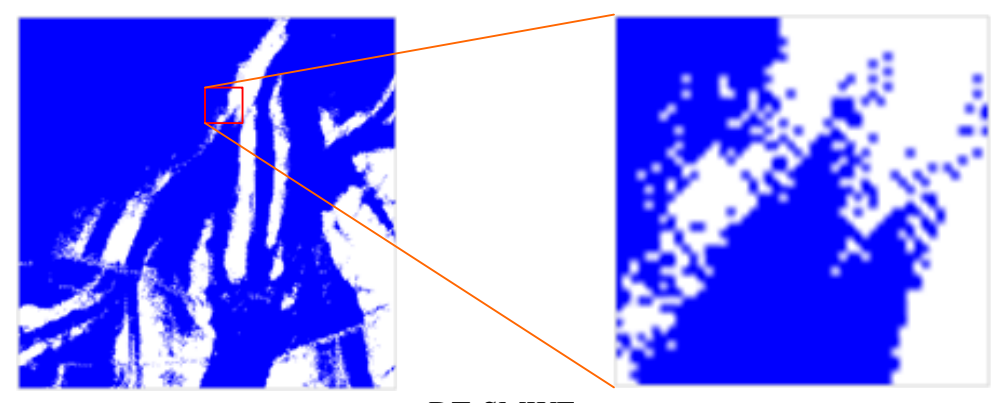

DT-SMWF

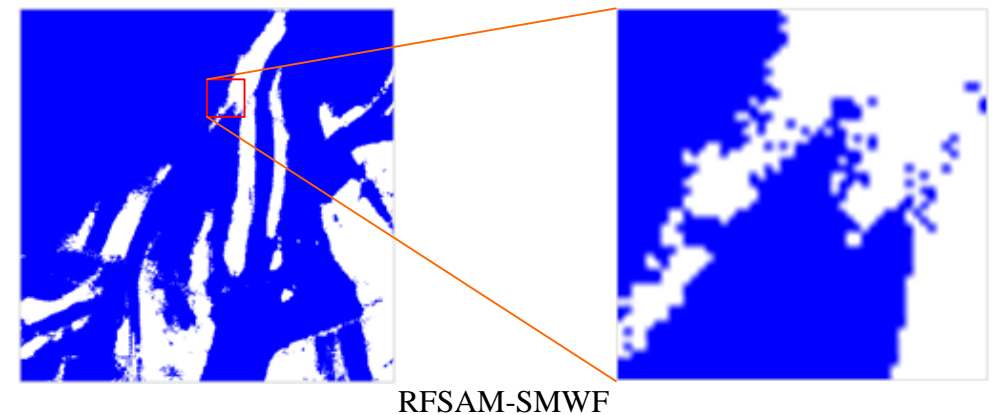

Figure 4. Mapping results of different SMWF methods for East Dongting Lake Wetland (scale = 5). 


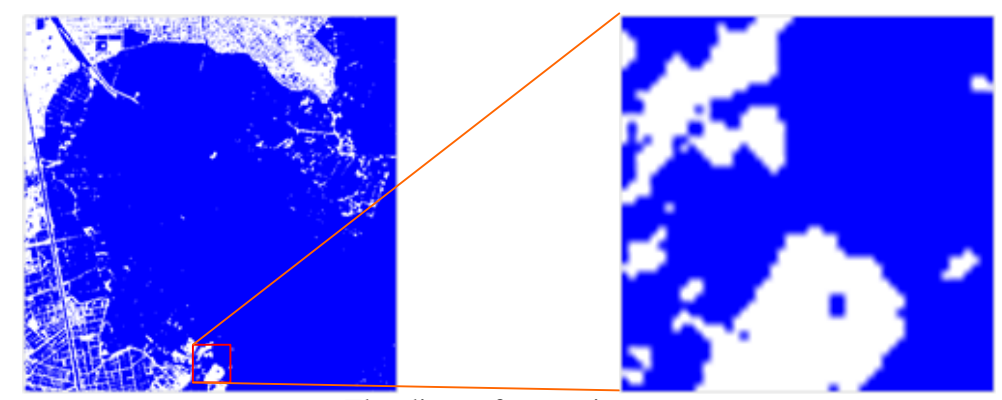

Flooding reference image
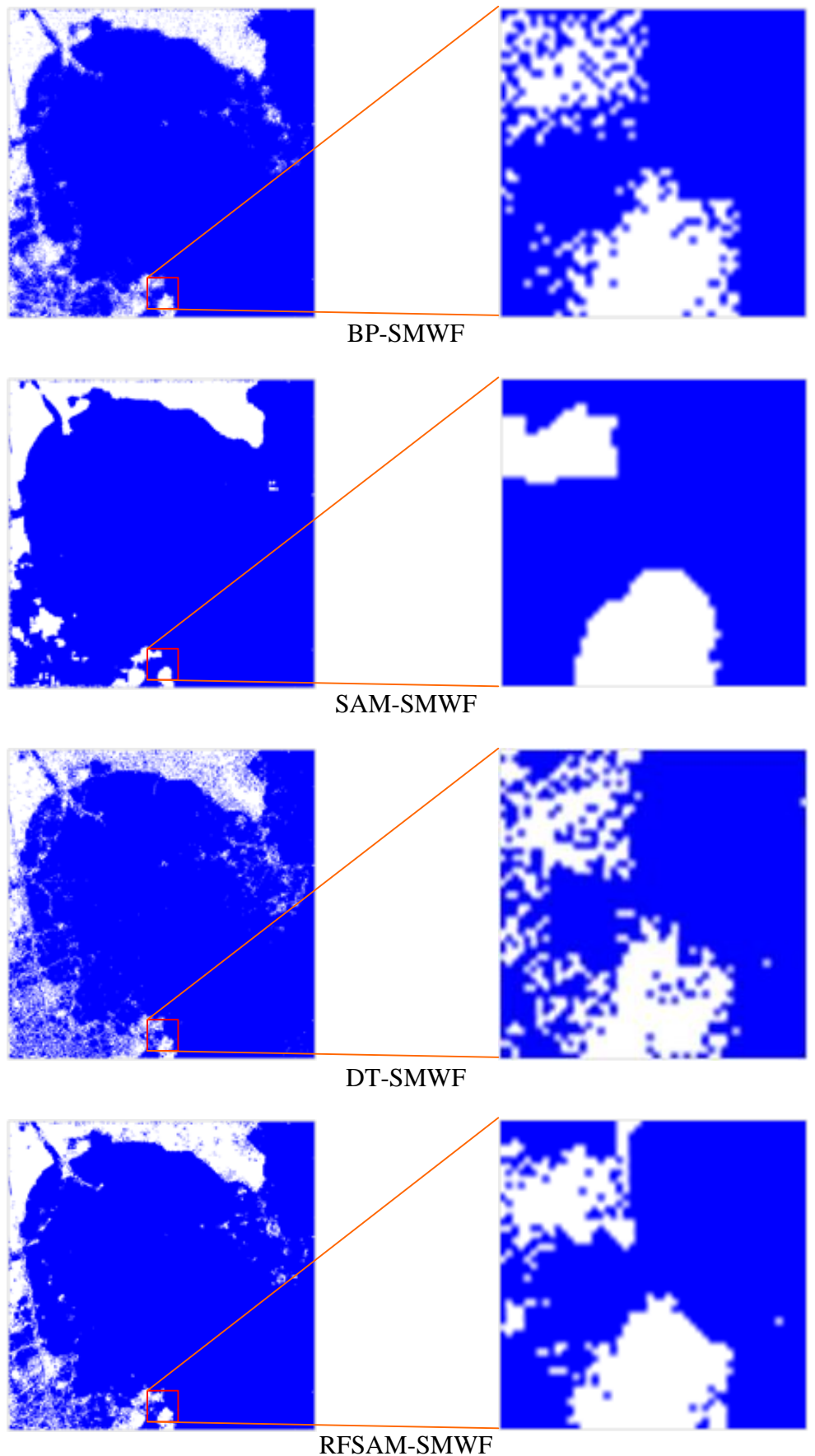

Figure 5. Mapping results of different SMWF methods for Honghu Wetland (scale = 5). 
Table 2. Quantitative metrics for each SMWF method.

\begin{tabular}{ccccccccc}
\hline \multirow{2}{*}{ Methods } & \multicolumn{4}{c}{ East Dongting Lake Wetland } & \multicolumn{4}{c}{ Honghu Wetland } \\
\cline { 2 - 9 } & OA (\%) & KC & APA (\%) & AUA (\%) & OA (\%) & KC & APA (\%) & AUA (\%) \\
\hline BP-SMWF & 72.8 & 0.454 & 72.7 & 72.7 & 70.1 & 0.393 & 69.6 & 69.7 \\
SAM-SMWF & 75.8 & 0.513 & 75.5 & 76.1 & 73.3 & 0.447 & 71.9 & 73.7 \\
DT-SMWF & 75.5 & 0.508 & 75.4 & 75.4 & 70.2 & 0.397 & 69.9 & 69.8 \\
RFSAM-SMWF & 80.2 & 0.601 & 80.0 & 80.2 & 76.7 & 0.527 & 76.3 & 76.4 \\
\hline
\end{tabular}

\section{Discussion}

\subsection{Choosing the Number of Trees in RFSAM-SMWF}

The number of trees (NT) is a key parameter of random forests, which affects the classification ability of random forests and therefore affects the sub-pixel mapping accuracy of RFSAM-SMWF. The sub-pixel mapping accuracy of RFSAM-SMWF related to NT was analyzed. The Landsat 8 OLI image for East Dongting Lake Wetland in the case study was used with different NT values. The other parameters of RFSAM-SMWF were the same as those in the case study. The sub-pixel mapping accuracy of RFSAM-SMWF for each NT value is shown in Figure 6. It shows that the higher the NT value, the higher the OA value. The OA value increases from $79.8 \%$ to $80.5 \%$ when the NT value rises from 10 to 50. The KC, APA, and AUA values have a similar increasing trend as the OA value. That is because random forests are a combination of tree predictors and high NT value increases the classification ability of random forests, which is favorable to increase the accuracy of RFSAM-SMWF. However, we can also see that the values of the evaluation indices have no obvious increase when the value of NT is larger than 40. Therefore, an NT value larger than 40 is unnecessary in this SMWF application.
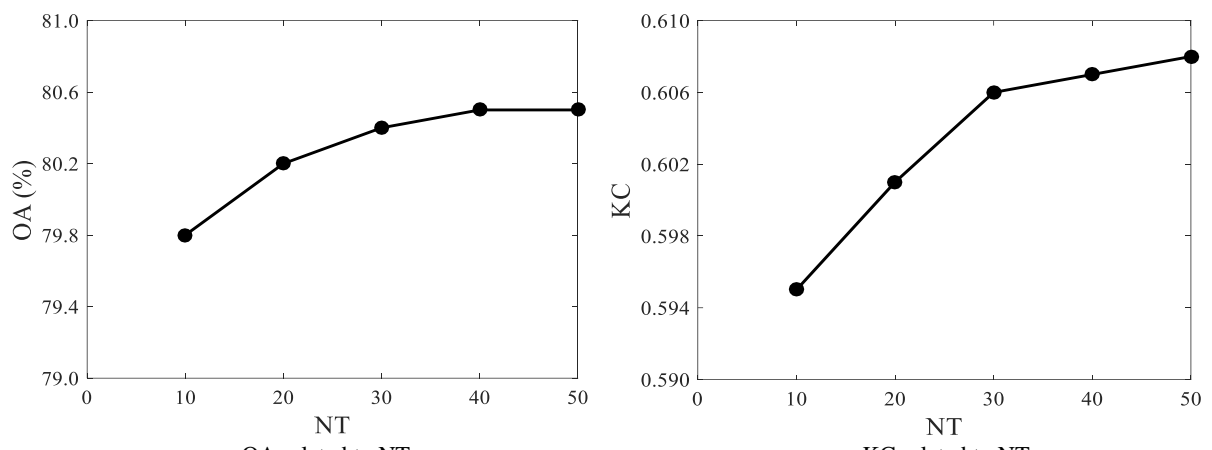

OA related to NT

$\mathrm{KC}$ related to NT
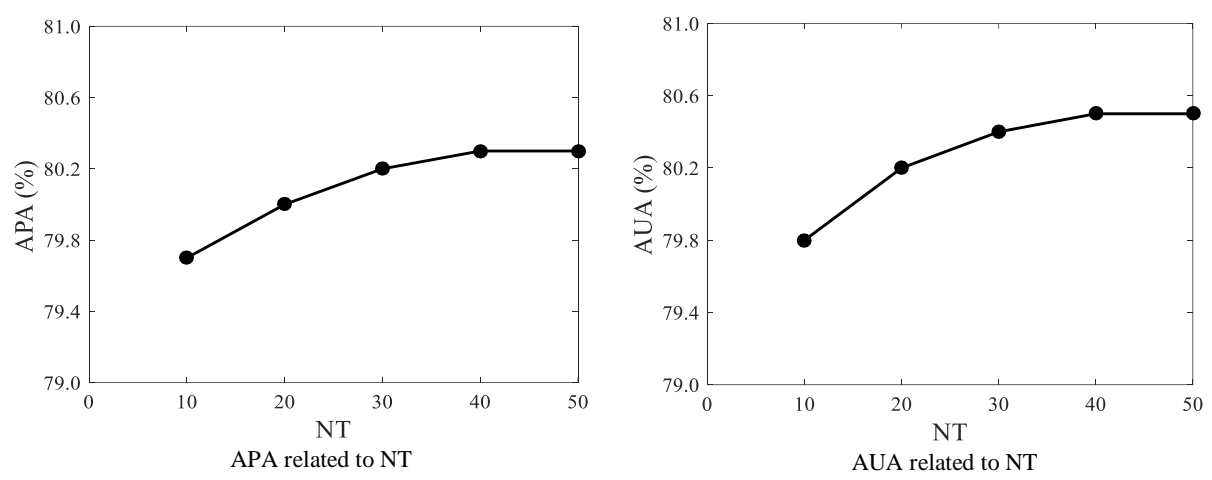

Figure 6. Sub-pixel mapping accuracy of RFSAM-SMWF related to the number of trees (NT) for East Dongting Lake Wetland, where NT represents the number of trees, OA represents overall accuracy, $\mathrm{KC}$ represents Kappa coefficient, APA represents average producer's accuracy, and AUA represents average user's accuracy. 


\subsection{Setting the Complexity Threshold in RFSAM-SMWF}

The complexity threshold (CT) determines the number of complex mixed pixels (NCMP) processed by random forests, which affects the sub-pixel mapping accuracy of RFSAM-SMWF. The sub-pixel mapping accuracy of RFSAM-SMWF related to CT was analyzed. The Landsat 8 OLI image for East Dongting Lake Wetland in the case study was used with different CT values. Other parameters of RFSAM-SMWF were the same as those in the case study. Sub-pixel mapping accuracy of RFSAM-SMWF for each CT value is shown in Table 3, where NMP represents the number of mixed pixels in the flooding fraction image and PCMP represents the percentage of complex mixed pixels. It indicates that the higher the CT value, the lower the OA value. The OA value decreases from $80.7 \%$ to $76.9 \%$ when the $\mathrm{CT}$ value rises from 0.05 to 0.50 . The $\mathrm{KC}, \mathrm{APA}$, and AUA values have a similar decreasing trend as the OA value. That is because a high CT value decreases the number of mixed pixels processed by random forests and increases the number of mixed pixels processed by SAM. Although SAM has several advantages in terms of its simplicity and its explicit physical meanings [8], random forests have a stronger ability to obtain satisfactory SMWF results than SAM in the complex situation.

Table 3. Sub-pixel mapping accuracy of RFSAM-SMWF related to the complexity threshold (CT) for East Dongting Lake Wetland, where NCMP represents the number of complex mixed pixels, NMP represents the number of mixed pixels in the flooding fraction image, and PCMP represents the percentage of complex mixed pixels.

\begin{tabular}{cccccccc}
\hline CT & NCMP & NMP & PCMP (\%) & OA (\%) & KC & APA (\%) & AUA (\%) \\
\hline 0.05 & 1423 & 2058 & 69.1 & 80.7 & 0.613 & 80.6 & 80.7 \\
0.10 & 991 & 2058 & 48.2 & 80.2 & 0.601 & 80.0 & 80.2 \\
0.20 & 563 & 2058 & 27.4 & 78.9 & 0.575 & 78.6 & 79.0 \\
0.50 & 177 & 2058 & 8.6 & 76.9 & 0.534 & 76.5 & 77.1 \\
\hline
\end{tabular}

\subsection{Evaluating SMWF Algorithms with Data from a Large Area}

One large area located in East Dongting Lake Wetland was selected for further evaluation and analysis of the SMWF algorithms. The materials of the large area $(3500 \times 2000$ pixels $)$ and the mapping results of different methods are shown in Figure 7. The location is shown in a color composite (R6G3B2) Landsat 8 OLI image. The reference image was obtained from the corresponding Landsat image using mNDWI. The parameters of the four SMWF methods are the same as those in the case study. Table 4 shows the performances of BP-SMWF, SAM-SMWF, DT-SMWF, and RFSAM-SMWF in terms of the accuracy obtained from the large area. RFSAM-SMWF obtained the best flooding mapping results among the four SMWF methods in the large area. It exhibited the highest values of OA, Kappa, APA, and AUA. For example, the OA values of BP-SMWF, SAM-SMWF, DT-SMWF, and RFSAM-SMWF are $74.1 \%, 78.3 \%, 79.4 \%$, and $82.9 \%$. SMWF is a complex multidimensional issue because there could be many possible sub-pixel distributions in the mixed pixels. Random forests can generate an internal unbiased estimate of the generalization error as the forest building progresses and do not overfit as more trees are added into the forest [20]. Therefore, RFSAM-SMWF can obtain satisfactory results in the complex situation and is suitable for both small and large areas. 


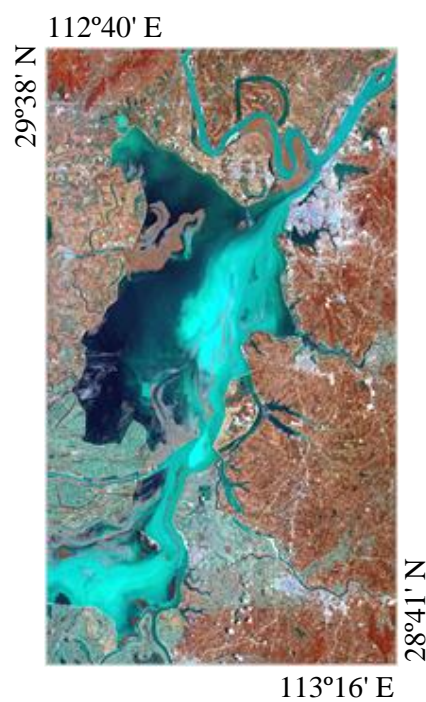

Landsat 8 OLI image (R6G3B2)

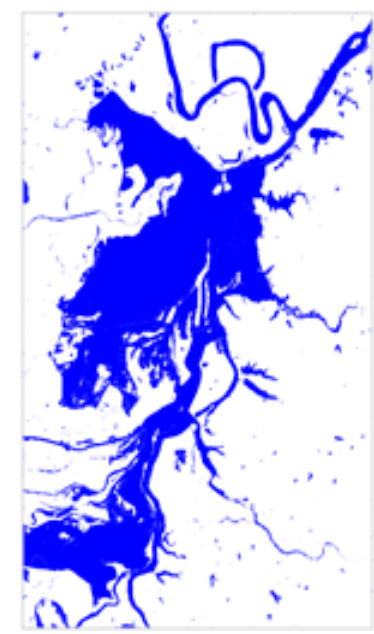

BP-SMWF

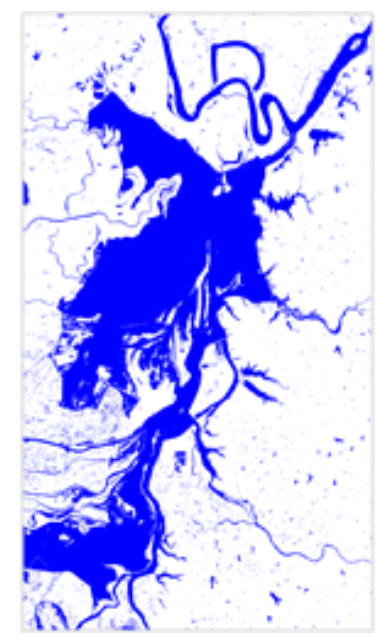

DT-SMWF

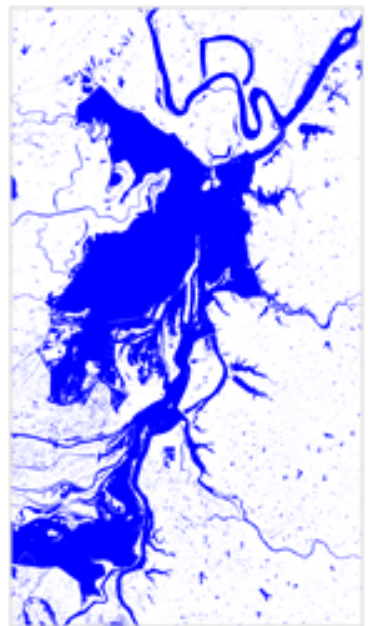

Flooding reference image

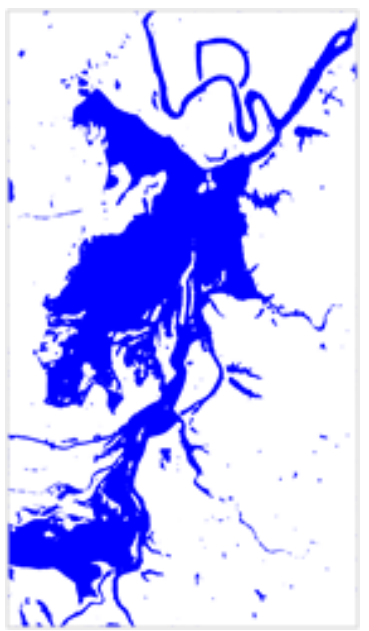

SAM-SMWF

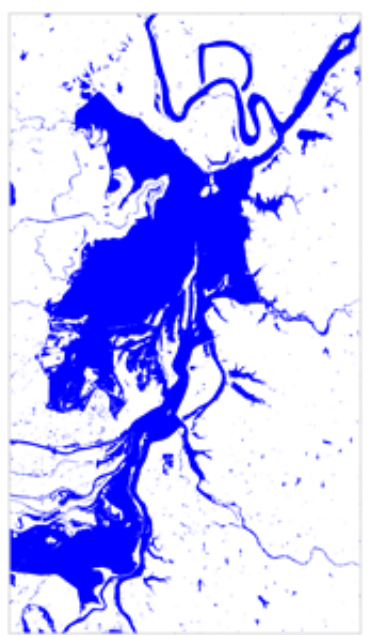

RFSAM-SMWF

Figure 7. Materials of a large area $(3500 \times 2000$ pixels $)$ located in East Dongting Lake Wetland and the mapping results of different methods (scale $=5$ ). 
Table 4. Quantitative comparisons of the performances of different methods in the large area $(3500 \times 2000$ pixels $)$.

\begin{tabular}{ccccc}
\hline \multirow{2}{*}{ Methods } & \multicolumn{4}{c}{ East Dongting Lake Wetland } \\
\cline { 2 - 5 } & OA (\%) & Kappa & APA (\%) & AUA (\%) \\
\hline BP-SMWF & 74.1 & 0.397 & 68.8 & 71.6 \\
SAM-SMWF & 78.3 & 0.492 & 73.1 & 77.3 \\
DT-SMWF & 79.4 & 0.542 & 76.8 & 77.4 \\
RFSAM-SMWF & 82.9 & 0.606 & 79.1 & 82.1 \\
\hline
\end{tabular}

\section{Conclusions}

Wetland flooding is necessary for the flora and fauna of wetlands and is important for the recovery of the wetland biodiversity. In this study, a novel method called RFSAM-SMWF was proposed to improve the mapping accuracy of wetland flooding at a sub-pixel scale from remote sensing images. The RM-SMWF algorithm was first developed, and the comprehensive complexity index of a mixed pixel was formulated. Then the RFSAM-SMWF algorithm was developed. The RFSAM-SMWF algorithm was evaluated using Landsat 8 OLI images of two wetlands of international importance included in the Ramsar List. RFSAM-SMWF was compared to three other SMWF methods, and it consistently achieved more accurate sub-pixel mapping results in terms of visual and quantitative assessments in the two wetlands. For example, the OA values of BP-SMWF, SAM-SMWF, DT-SMWF, and RFSAM-SMWF are $72.8 \%, 75.8 \%, 75.5 \%$, and $80.2 \%$ for East Dongting Lake Wetland, respectively. And the OA values of BP-SMWF, SAM-SMWF, DT-SMWF, and RFSAM-SMWF are 70.1\%, 73.3\%, 70.2\%, and $76.7 \%$ for Honghu Wetland, respectively. RFSAM-SMWF can obtain satisfactory results in the complex situation and is suitable for both small and large areas. The results of this study improve the mapping accuracy of wetland flooding from medium-low spatial resolution remote sensing images and therefore benefit the environmental studies of wetlands.

Author Contributions: Data curation, L.L., K.S., R.L., C.H. and B.L.; Methodology, L.L.; Supervision, Y.C., T.X. and L.M.; Writing-original draft, L.L.

Funding: This research was funded by the National Key Research and Development Program of China, grant number [2018YFC0407804], and the National Natural Science Foundation of China, grant number [41371343].

Acknowledgments: The authors would like to thank Susan Cuddy at the Commonwealth Scientific and Industrial Research Organisation (CSIRO) for her helpful discussions and constructive suggestions.

Conflicts of Interest: The authors declare no conflict of interest.

\section{References}

1. Sarkar, U.K.; Borah, B.C. Flood plain wetland fisheries of India: With special reference to impact of climate change. Wetl. Ecol. Manag. 2018, 26, 1-15.

2. Ramsar. The Importance of Wetlands. Available online: https://www.ramsar.org (accessed on 5 December 2018).

3. Chen, Y.; Liu, R.; Barrett, D.; Gao, L.; Zhou, M.; Renzullo, L.; Emelyanova, I. A spatial assessment framework for evaluating flood risk under extreme climates. Sci. Total Environ. 2015, 538, 512-523. [CrossRef]

4. Huang, C.; Chen, Y.; Zhang, S.; Wu, J. Detecting, extracting, and monitoring surface water from space using optical sensors: A review. Rev. Geophys. 2018, 56, 333-360. [CrossRef]

5. Li, L.; Chen, Y.; Xu, T.; Liu, R.; Shi, K.; Huang, C. Super-resolution mapping of wetland inundation from remote sensing imagery based on integration of back-propagation neural network and genetic algorithm. Remote Sens. Environ. 2015, 164, 142-154. [CrossRef]

6. Huang, C.; Chen, Y.; Zhang, S.; Li, L.; Shi, K.; Liu, R. Spatial downscaling of Suomi NPP-VIIRS image for lake mapping. Water 2017, 9, 834. [CrossRef]

7. Wu, S.; Ren, J.; Chen, Z.; Jin, W.; Liu, X.; Li, H.; Pan, H.; Guo, W. Influence of reconstruction scale, spatial resolution and pixel spatial relationships on the sub-pixel mapping accuracy of a double-calculated spatial attraction model. Remote Sens. Environ. 2018, 210, 345-361. [CrossRef] 
8. Lu, L.; Huang, Y.; Di, L.; Hang, D. A new spatial attraction model for improving subpixel land cover classification. Remote Sens. 2017, 9, 360. [CrossRef]

9. Wu, K.; Du, Q.; Hu, X.; Wang, X. Sub-Pixel mapping based on MAP model and spatial attraction theory for remotely sensed image. IEEE Access. 2017, 5, 25126-25132. [CrossRef]

10. Li, L.; Xu, T.; Chen, Y. Improved urban flooding mapping from remote sensing images using generalized regression neural network-based super-resolution algorithm. Remote Sens. 2016, 8, 625. [CrossRef]

11. Arun, P.V.; Buddhiraju, K.M.; Porwal, A. CNN based sub-pixel mapping for hyperspectral images. Neurocomputing 2018, 311, 51-64. [CrossRef]

12. Tiwari, L.K.; Sinha, S.K.; Saran, S.; Tolpekin, V.A.; Raju, P.L.N. Markov random field-based method for super-resolution mapping of forest encroachment from remotely sensed ASTER image. Geocartogr. Int. 2016, 31, 428-445. [CrossRef]

13. He, D.; Zhong, Y.; Feng, R.; Zhang, L. Spatial-temporal sub-pixel mapping based on swarm intelligence theory. Remote Sens. 2016, 8, 894. [CrossRef]

14. Li, L.; Chen, Y.; Yu, X.; Liu, R.; Huang, C. Sub-pixel flood inundation mapping from multispectral remotely sensed images based on discrete particle swarm optimization. ISPRS J. Photogramm. Remote Sens. 2015, 101, 10-21. [CrossRef]

15. Nami, S.; Shajari, M. Cost-sensitive payment card fraud detection based on dynamic random forest and k-nearest neighbors. Expert Syst. Appl. 2018, 110, 381-392. [CrossRef]

16. Paul, A.; Mukherjee, D.P.; Das, P.; Gangopadhyay, A.; Chintha, A.R.; Kundu, S. Improved random forest for classification. IEEE Trans. Image Process. 2018, 27, 4012-4024. [CrossRef]

17. Zhang, H.; Li, Q.; Liu, J.; Shang, J.; Du, X.; McNairn, H.; Champagne, C.; Dong, T.; Liu, M. Image classification using RapidEye data: Integration of spectral and textual features in a random forest classifier. IEEE J. Sel. Top. Appl. Earth Observ. Remote Sens. 2017, 10, 5334-5349. [CrossRef]

18. Xia, X.; Togneri, R.; Sohel, F.; Huang, D. Random forest classification based acoustic event detection utilizing contextual-information and bottleneck features. Pattern Recogn. 2018, 81, 1-13. [CrossRef]

19. Breiman, L. Random forests. Mach. Learn. 2001, 45, 5-32. [CrossRef]

20. Breiman, L.; Cutler, A. Random Forests. Available online: https://www.stat.berkeley.edu/ \{\}breiman/ RandomForests/cc_home.htm (accessed on 10 December 2018).

21. Brokamp, C.; Jandarov, R.; Hossain, M.; Ryan, P. Predicting daily urban fine particulate matter concentrations using a random forest model. Environ. Sci. Technol. 2018, 52, 4173-4179. [CrossRef]

22. Araki, S.; Shima, M.; Yamamoto, K. Spatiotemporal land use random forest model for estimating metropolitan $\mathrm{NO}_{2}$ exposure in Japan. Sci. Total Environ. 2018, 634, 1269-1277. [CrossRef]

23. Ouallouche, F.; Lazri, M.; Ameur, S. Improvement of rainfall estimation from MSG data using random forests classification and regression. Atmos. Res. 2018, 211, 62-72. [CrossRef]

24. Zimmer, V.A.; Glocker, B.; Hahner, N.; Eixarch, E.; Sanroma, G.; Gratacos, E.; Rueckert, D.; Ballester, M.A.G.; Piella, G. Learning and combining image neighborhoods using random forests for neonatal brain disease classification. Med. Image Anal. 2017, 42, 189-199. [CrossRef]

25. Su, X.; Pena, A.T.; Liu, L.; Levine, R.A. Random forests of interaction trees for estimating individualized treatment effects in randomized trials. Stat. Med. 2018, 37, 2547-2560. [CrossRef]

26. Assouline, D.; Mohajeri, N.; Scartezzini, J.L. Large-scale rooftop solar photovoltaic technical potential estimation using Random Forests. Appl. Energy 2018, 217, 189-211. [CrossRef]

27. Renno, C.; Petito, F. Triple junction cell temperature evaluation in a CPV system by means of a Random-Forest model. Energy Convers. Manag. 2018, 169, 124-136. [CrossRef]

28. Wang, Z.; Wang, Y.; Zeng, R.; Srinivasan, R.S.; Ahrentzen, S. Random Forest based hourly building energy prediction. Energy Build. 2018, 171, 11-25. [CrossRef]

29. Wang, B.; Gao, L.; Juan, Z. Travel mode detection using GPS data and socioeconomic attributes based on a random forest classifier. IEEE Trans. Intell. Transp. Syst. 2018, 19, 1547-1558. [CrossRef]

30. Kuang, X.; Fu, W.; Yang, L. Real-time detection and recognition of road traffic signs using MSER and random forests. Int. J. Online Eng. 2018, 14, 34-51. [CrossRef]

31. Behrens, C.; Pierdzioch, C.; Risse, M. Testing the optimality of inflation forecasts under flexible loss with random forests. Econ. Model. 2018, 72, 270-277. [CrossRef]

32. Houborg, R.; McCabe, M.F. A hybrid training approach for leaf area index estimation via Cubist and random forests machine-learning. ISPRS J. Photogramm. Remote Sens. 2018, 135, 173-188. [CrossRef] 
33. Pullanagari, R.R.; Kereszturi, G.; Yule, I. Integrating airborne hyperspectral, topographic, and soil data for estimating pasture quality using recursive feature elimination with random forest regression. Remote Sens. 2018, 10, 1117. [CrossRef]

34. Liu, W.; Yang, J.; Li, P.; Han, Y.; Zhao, J.; Shi, H. A novel object-based supervised classification method with active learning and random forest for PolSAR imagery. Remote Sens. 2018, 10, 1092. [CrossRef]

35. Mertens, K.C.; De Baets, B.; Verbeke, L.P.C.; De Wulf, R.R. A sub-pixel mapping algorithm based on sub-pixel/pixel spatial attraction models. Int. J. Remote Sens. 2006, 27, 3293-3310. [CrossRef]

36. Ramsar. Wetlands of International Importance (Ramsar Sites). Available online: https://www.ramsar.org (accessed on 12 December 2018).

37. Chetan, M.A.; Dornik, A.; Urdea, P. Analysis of recent changes in natural habitat types in the Apuseni Mountains (Romania), using multi-temporal Landsat satellite imagery (1986-2015). Appl. Geogr. 2018, 97, 161-175. [CrossRef]

38. Markogianni, V.; Kalivas, D.; Petropoulos, G.P.; Dimitriou, E. An appraisal of the potential of Landsat 8 in estimating Chlorophyll-a, Ammonium Concentrations and other water quality indicators. Remote Sens. 2018, 10, 1018. [CrossRef]

39. Ge, Y.; Chen, Y.; Li, S.; Jiang, Y. Vectorial boundary-based sub-pixel mapping method for remote-sensing imagery. Int. J. Remote Sens. 2014, 35, 1756-1768. [CrossRef]

40. The MathWorks, Inc. Available online: https://ww2.mathworks.cn/help/ (accessed on 15 December 2018).

41. Cabral, A.I.R.; Silva, S.; Silva, P.C.; Vanneschi, L.; Vasconcelos, M.J. Burned area estimations derived from Landsat ETM plus and OLI data: Comparing genetic programming with maximum likelihood and classification and regression trees. ISPRS J. Photogramm. Remote Sens. 2018, 142, 94-105. [CrossRef]

42. Chen, J.; Du, P.; Wu, C.; Xia, J.; Chanussot, J. Mapping urban land cover of a large area using multiple sensors multiple features. Remote Sens. 2018, 10, 872. [CrossRef]

(C) 2019 by the authors. Licensee MDPI, Basel, Switzerland. This article is an open access article distributed under the terms and conditions of the Creative Commons Attribution (CC BY) license (http://creativecommons.org/licenses/by/4.0/). 